\title{
Broken Heart (Takotsubo) Syndrome Triggered by SARS-CoV-2
}

\author{
Josef Finsterer ${ }^{1}$ (D) \\ Accepted: 9 December 2020 / Published online: 12 January 2021 \\ (C) The Author(s), under exclusive licence to Springer Nature Switzerland AG part of Springer Nature 2021
}

Letter to the Editor

With interest, I read the review article by Singh et al. about 12 SARS-CoV-2 positive patients with COVID-19 who developed broken heart (Takotsubo) syndrome (TTS) in the course of the viral infection [1]. It was concluded that COVID-19related TTS is particularly prevalent in elder females and that direct myocardial injury, inflammation, or stress may lead to the occurrence of COVID-19-related TTS [1]. I have the following comments and concerns.

The authors mention that their study is the first systematic review about COVID-19-related TSS [1], but in the results section, they indicate that 5 papers were excluded from the evaluation because they were review articles [1]. We should be told if these 5 articles were reviews about COVID-19related TTS.

To underscore how fast the literature about COVID-19related TTS is growing, 12 patients had been reported as per September 13, 38 patients as per October 6 [2], at least 45 patients as per October 15, and at least 50 patients as per the end of November 2020.

The authors mention that TTS in COVID-19 patients is caused by the cytokine storm [1]. They consider that the cytokine storm aroused by the infection could trigger TTS, particularly among patients without an evident trigger. However, no evidence for this speculation is provided. According to expert opinions, TTS is due to physical or mental stress in one-third of the cases each [3]. Thus, the cause of TTS remains speculative in at least one-third of the cases. To unravel the cause of TTS in the one-third without an obvious trigger, it is not only crucial to carefully review the individual and drug history, the socioeconomic context, and the personal circumstances at the time TTS evolved but also to investigate if an

This article is part of the Topical Collection on Covid-19

Josef Finsterer

fifigs1@yahoo.de

1 Klinik Landstrasse, Messerli Institute, Postfach 20, 1180 Vienna, Austria overshooting immune response could trigger transient heart failure.

The authors propose myocarditis as a pathophysiological factor in the development of TTS [1]. It would be interesting to know if myocarditis in patients with COVID-19-related TTS was only suspected, confirmed upon cardiac MRI with contrast medium, or if myocarditis has been diagnosed in any of the included patients upon endomyocardial biopsy. According to currently available data, myocarditis has been definitively diagnosed in only one patient with COVID-19related TTS so far [4]. However, there is not only evidence from the literature that myocarditis may mimic TTS but that myocarditis may indeed cause TTS in some cases [5].

Overall, the interesting review about COVID-19-related TTS by Singh et al. has limitations as outlined above. To elucidate the pathophysiology of COVID-19-related TTS, it is crucial to carefully take the individual history which should be backed up by close relatives or friends. So far, there is no evidence that the infection with SARS-CoV-2 per se triggers TTS but rather the mental or physical stress associated with the infection.

Author Contribution JF: design, literature search, discussion, first draft, critical comments.

\section{Compliance with Ethical Standards}

The study was approved by the institutional review board.

Conflict of Interest The author declares that he has no conflicts of interest.

Informed Consent Informed consent was obtained.

\section{References}

1. Singh S, Desai R, Gandhi Z, Fong HK, Doreswamy S, Desai V, et al. Takotsubo syndrome in patients with COVID-19: a systematic review of published cases. SN Compr Clin Med. 2020;6:1-7. https:// doi.org/10.1007/s42399-020-00557-w.

2. Finsterer J, Stöllberger C. SARS-CoV-2 triggered Takotsubo in 38 patients. J Med Virol. 2020. https://doi.org/10.1002/jmv.26581. 
3. Okura H. Update of takotsubo syndrome in the era of COVID-19. J Cardiol. 2020 :S0914-5087(20)30331-2. https://doi.org/10.1016/j. jjcc.2020.10.004.

4. Sala S, Peretto G, Gramegna M, Palmisano A, Villatore A, Vignale $\mathrm{D}$, et al. Acute myocarditis presenting as a reverse Tako-Tsubo syndrome in a patient with SARS-CoV-2 respiratory infection. Eur Heart J. 2020;41:1861-2. https://doi.org/10.1093/eurheartj/ehaa286.
5. Cusmà-Piccione M, Longobardo L, Oteri A, Manganaro R, Di Bella $\mathrm{G}$, Carerj S, et al. Takotsubo cardiomyopathy: queries of the current era. J Cardiovasc Med (Hagerstown). 2018;19:624-32. https://doi. org/10.2459/JCM.0000000000000707.

Publisher's Note Springer Nature remains neutral with regard to jurisdictional claims in published maps and institutional affiliations. 